\title{
A multifaceted approach to prevention of delirium on intensive care
}

\author{
James Hanison, Daniel Conway
}

Manchester Royal Infirmary

\begin{abstract}
Delirium is a frequent complication of critical illness with an increased risk of death which is difficult to treat effectively. We describe a seven year quality improvement programme that aimed to reduce rates of delirium on intensive care (ICU). We completed two PDSA programmes with a number of changes including alterations to sedation practice and environmental changes to the ICU itself.
\end{abstract}

Rates of delirium reduced from $70 \%$ to $44 \%$ after the first cycle of change. Rates of delirium were further reduced to $29 \%$ after the second cycle of change. The rates are now lower than predicted by the validated prediction tool PRE-DELIRIC.

This project demonstrates that a multifaceted approach to prevention of delirium on ICU can deliver sustained reductions in rates of delirium. The impact of the second cycle of change was less than the first, suggesting that further reductions may prove more challenging.

\section{Problem}

Delirium is a frequently occurring event in critical care units that incurs an independent risk for poor short and long term outcomes for patients. It is can be difficult to diagnose delirium and, when delirium is identified, it can be difficult to treat with many drug therapies largely ineffective.

In order to reduce rates of delirium in critical care, a multifaceted approach is required with a particular emphasis on nonpharmacological treatments.

\section{Background}

Delirium may be described as a disturbance of consciousness, attention, cognition, and perception which develops over a short period of time (usually hours to days) and tends to fluctuate during the course of the day.[1] Prevalence of this condition in UK ICUs using current screening tools has been estimated at $45 \%$ of unplanned admissions.[2] Delirium is an independent risk factor for mortality; for every day of delirium on ICU, there is an additional $10 \%$ relative risk of death at 1 year.[3]

There are a number of risk factors for the development of delirium on ICU which are frequently occurring. These include:

- Increasing age

- Severity of illness ie increasing APACHE II score

- Coma (greater risk for coma that is not purely drug induced)

- Infection

- Opiate use (dose dependent risk)

- Use of sedatives

- Raised urea

- Unplanned admission[4]

A number of trials have been performed to identify whether certain interventions reduce the risk of delirium; many of these trials have focused on drug treatments and few have demonstrated any benefit.[5] It was anticipated that a number of interventions would be required to reduce the rates of delirium.

\section{Baseline measurement}

The project started in 2007 by measuring delirium across our critical care units (both ICU and HDU). We noted whether a patient had delirium at the first assessment; delirium at any point during their critical care stay and the number of positive assessments that were made. Data were collected about known risk factors for delirium. The incidence of delirium in critical care was 22/55 (40\%) but for level 3 ICU patients the incidence was 16/23 (70\%).

\section{Design}

Manchester Royal Infirmary has a 38 bed mixed general adult critical care unit, which has incrementally increased in size since 2007 (23 beds). A number of clinical, environmental, and cultural changes were made to reduce delirium rates during the course of the project. A large number of clinical practices were changed, including the reduction in use of deliriogenic drugs such as benzodiazepines, long acting opioids, the targeting of lighter levels of sedation and the routine use of daily sedation breaks. The environment has also changed; the unit has expanded and moved into a larger and more open plan area with better access to natural light and more space at the bedside. Cultural changes also occurred as staff engaged with issue of delirium on ICU.

Throughout the project we used the Richmond Agitation and Sedation Score (RASS) once sedative agents were ceased $>24$ hours and Confusion Assessment Method for the Intensive Care Unit (CAM-ICU), a validated tool for assessing ICU delirium.[6]

\section{Strategy}


As the majority of delirium occurred in ICU patients who had been sedated and mechanically ventilated, we targeted this group for the improvement project. During this period we introduced modules on recognition and management of delirium into our existing teaching programmes for medical, nursing, and Allied Health Professionals. Consultant intensivists and critical care pharmacists championed the use of short acting sedative agents, daily sedation breaks, encouraging lighter levels of sedation and assessment for delirium. This involved the re-design of drug booklets, guidelines for the use of a very short acting opioid (remifentanil), and redesign of the nursing charts. Non-pharmacological management of delirium including electronic communication aids were encouraged alongside pharmacological management, which was largely reserved for hyperactive delirium.

PDSA cycle 2 - 2012-2014

During this period, we refurbished the critical care areas, allowing more space between beds, increased natural light, and placed clocks within patient's view. Staff training was enhanced by feedback from our ICU follow-up team of stories from patients who had experienced delirium. We introduced dexmedetomidine, a sedative agent with low associated levels of delirium. The daily medical and nursing charts prompted assessment of delirium. We audited the incidence of delirium using CAM-ICU alongside the PRE-DELIRIC risk assessment tool.[4]

\section{Results}

The initial audit highlighted high rates of delirium; $65 \%$ of 23 ICU patients had delirium at first assessment and $70 \%$ of patients had delirium at some point during their ICU stay. After the first PDSA cycle, delirium rates were much improved; $16 \%$ of 85 ICU patients had delirium at first assessment and $44 \%$ of patients had delirium at some point. The third cycle demonstrated a sustained effect with consistently low rates of delirium; $15 \%$ of 42 patients had delirium at first assessment and $29 \%$ of patients had delirium at some point, this compared to the PRE-DELIRIC predicted average rate of $39 \%$.

The rates of patients who were being exposed to deliriogenic drugs in the first audit were high, with nearly all patients on such drugs $(87 \%)$. This number had been reduced to $70 \%$ by completion of the third audit. In the initial audit the median number of positive assessments per patient with delirium at any time was 5 (range 1 to 12), this fell to 2 ( 1 to 12 ) in the second audit, and 3 (range 1 to 14 ) in the final audit.

\section{Lessons and limitations}

The rates of delirium were much improved after the first PDSA cycle. There was a less marked reduction in the rates of delirium after the following PDSA cycle. Although the following audit demonstrated that the improvement after the first cycle was maintained, it appears that a plateau had been reached. more difficult to improve upon. This is unsurprising, as many of the risk factors for delirium such as age, pre-existing cognitive decline, psychiatric co-morbidity, and severity of disease are not modifiable.

The CAM-ICU tool is well validated in similar populations of critically ill patients. The tool has limitations as it cannot be applied to every patient, it requires careful clinical judgement to distinguish between hypoactive delirium and residual sedation and most assessments were performed during daytime rather than night-time when delirious episodes can occur.

\section{Conclusion}

Although delirium on ICU is regarded as a condition that is difficult to prevent and treat, we have demonstrated that it is possible to reduce rates of delirium on the ICU. We have also demonstrated that it is possible to sustain these improvements over a period of time. Delirium on ICU cannot be managed by drug therapy alone; in order to produce sustained reduction in rates, a multimodal approach is most effective.

\section{References}

1. American Psychiatric Association. Diagnostic and Statistical Manual of Mental Disorders Fourth Edition, text revision. Washington, DC: American Psychiatric Association; 2000.

2. Page VJ, Navarange S, Gama S, et al. Routine delirium monitoring in a UK critical care unit. Crit Care 2009; 13: R16.

3. Pisani MA, Kong SY, KasI SV, et al. Days of delirium are associated with 1-year mortality in an older intensive care unit population. Am J Respir Crit Care Med. 2009; 180(11): 1092-7.

4. van den Boogaard M, Pickkers $P$, Slooter AJ, et al. Development and validation of PRE-DELIRIC (PREdiction of DELIRium in ICu patients) delirium prediction model for intensive care patients: observational multicentre study. BMJ 2012; 344: e420.

5. Al-Qadheeb NS, Balk EM, Fraser GL, et al. Randomized ICU trials do not demonstrate an association between interventions that reduce delirium duration and short-term mortality: a systematic review and meta-analysis. Crit Care Med. 2014; 42(6): 1442-54.

6. Ely EW, Inouye SK, Bernard GR, et al. Delirium in mechanically ventilated patients: validity and reliability of the Confusion Assessment Method for the Intensive Care Unit (CAM-ICU). JAMA 2001; 286:2703-10.

\section{Declaration of interests}

Dr James Hanison has no interests to declare.

Dr Daniel Conway has received honoraria from Orion Pharma and Glaxo-Smith-Kline.

The General Adult Critical Care Unit at Manchester Royal Infirmary 


\section{BMJ Quality Improvement Reports}

received an unrestricted educational grant from Orion Pharma during this period.

\section{Acknowledgements}

The assistance of the dedicated staff at Manchester Royal Infirmary Critical Care Unit is gratefully acknowledged.

In particular: Keyur Acharya, Richard Clarke, Saadia Umar, Hannah Lees and Adam Jones.

\section{Ethical approval}

This project represents an evaluation of an established service and changes made in order to improve the service by aligning it to already proven best practice. The project did not at any point attempt to answer a hypothesis by experimentation and, as such, does not constitute research. It is for this reason that ethical approval was not sought.

According to the policy activities that constitute research at Central Manchester Foundation Trust, this work met criteria for operational improvement activities exempt from ethics review.

Central Manchester Foundation Trust consider the following criteria for determining if improvement activities require ethics review:

Additional data or tissue samples being collected

- All data collected and assessments performed during the QI initiative formed part of the usual care of the patients. The critical care unit participates in the national ICNARC programme and therefore has a robust informatics and data collection system.

-Audit activity as part of the QI initiative was registered with, approved by and reviewed by the Clinical Audit Department of the hospital

-Follow Up data from national sources or primary care was not accessed

Administration of a novel drug and/or placebo

- Any new sedative medications introduced into critical care practice were subjected to scrutiny by the Medicines Management Committee of the hospital. All medications were licensed with the MHRA. The committee and the applicants did not consider the introduction of new agents to be clinical research or require review by the Local research Ethics committee

Comparing Different Clinical Practice

- The changes in practice encouraged during the quality improvement programme were in line with best practice and the UK NICE guideline on delirium CG 1032010.

-The work is primarily intended to improve local care, not provide generalizable knowledge in a field of inquiry. 